6. Upregulation of HOXC6 contributed to poor prognosis in colorectal cancer (figure $1 \mathrm{~K}$ )

Conclusions HOXC6 could upregulate DKK1, Wnt/b-catenin pathways and induce EMT which contributed to metastasis in colorectal cancer. Further, we will explore the mechanisms of how upregulation of DKK1 could activate Wnt/b-catenin pathway.

\section{IDDF2019-ABS-0342 EVALUATION OF BOWEL CANCER AWARENESS AMONG UNDERGRADUATE PHARMACY STUDENTS IN MALAYSIA AND PAKISTAN: A CROSS-SECTIONAL STUDY}

${ }^{1}$ Sohail Ahmad*, ${ }^{2}$ Rooha Ali, ${ }^{1}$ Muhammad Qamar, ${ }^{3}$ Faiz Ahmed Shaikh, ${ }^{4}$ Nauman Rahim Khan, 'Amutha Selvaraj. 'Faculty of Pharmacy, MAHSA University, Malaysia; ${ }^{2}$ College of Pharmacy, University of Sargodha, Pakistan; ${ }^{3}$ School of Pharmacy, Management and Science University, Malaysia; ${ }^{4}$ Faculty of Pharmacy, Gomal University, Pakistan

\subsection{6/gutjnl-2019-IDDFabstracts.82}

Background As a pharmacist-wannabes, the final year undergraduate pharmacy students must have adequate foundational cancer knowledge and training to educate the public regarding the identification of warning signs and risk factors of bowel cancer. This study was conducted to assess and compare the levels of bowel cancer awareness among pharmacy students in Malaysia and Pakistan.

Methods In this multi-centre cross-sectional study, a total of 174 final year undergraduate pharmacy students were enrolled from two Malaysian and two Pakistani universities. Post-approval from Cancer Research UK, a pre-validated Bowel Cancer Awareness Measure (CAM) was used. Bowel CAM consists of eight questions with a total of 26 items focusing on warning signs (10 items), delay in seeking medical help (1 item), bowel age (1 item), risk factors (11 items), bowel cancer screening programme (2 items), knowledge (1 item), age of first invitation (1 item), and confidence in detecting bowel symptom (1 item). The extracted data from the completed questionnaires were analysed descriptively and inferentially using the Statistical Package for the Social Sciences $\left(\mathrm{SPSS}^{(\mathrm{R})}\right)$, version 22.

Results The mean age of enrolled students was 22.78, $\mathrm{SD}=1.77$, and the majority of them were females $(n=107$, $61.5 \%)$, and Malaysians $(n=111,63.8 \%)$. The enrolled students possessed high and moderate levels of awareness regarding bowel cancer warning signs $(n=129,74.1 \%)$, and risk factors $(n=148,85.1 \%)$, respectively. The findings of an independent-samples t-test suggested that there was a statistically significant difference in warning signs awareness score in Malaysian $(M=24.13, \quad S D=2.67)$, and Pakistani students $[\mathrm{M}=22.57, \mathrm{SD}=3.43 ; \mathrm{t}(172)=3.332, \mathrm{p}=0.001]$; whereas, this difference was insignificant for the scores of risk factors $(\mathrm{M}=32.59, \mathrm{SD}=4.20), \quad[\mathrm{M}=31.30, \mathrm{SD}=4.65 ; \mathrm{t}(172)=1.875$, $\mathrm{p}=0.062]$. Moreover, a significant positive correlation was found between scores of warning signs and risk factors ( $\mathrm{r}=0.364, \mathrm{p}=0.001)$.

Conclusions Malaysian students showed a better score for Bowel CAM. Specialised periodic training sessions can be helpful to enhance awareness among these future pharmacists.

\section{IDDF2019-ABS-0343 INTRANUCLEAR MGP PROMOTES TUMOR PROGRESSION BY REGULATING JAK2I STAT5 PATHWAY AND INDICATES A POOR PROGNOSIS IN GASTRIC CANCER}

Lei Chen*, Mizhu Wang, Li Min, Shutian Zhang. Beijing Friendship Hospital, Capital Medical University, China

\subsection{6/gutjnl-2019-IDDFabstracts.83}

Background Matrix Gla protein (MGP) has been reported as an extracellular matrix protein with dysregulation in various types of malignancies. However, the regulatory function of MGP inside the nuclears of gastric cancer (GC) cells remains largely unknown. In this context, we aimed to investigate the intranuclear molecular mechanisms of MGP contributing to GC development and progression.

Methods MGP expression in GC was evaluated by immunohistochemistry and was further confirmed by two independent GEO datasets. Also, analysis of TCGA datasets illustrated its prognostic potential. Effects of MGP on GC cell proliferation, apoptosis, migration and invasion were all estimated. Luciferase reporter assays, immunoprecipitation, chromatin immunoprecipitation, et al were performed to figure out the underlying signaling pathways related to oncogenic effects of MGP.

Results We demonstrated a higher expression level of MGP in GC compared to the adjacent normal tissues, leading to a worse prognosis of GC patients (figure 1. A-C). MGP can promote proliferation, migration and invasion of GC cells while inhibit apoptosis (figure 1. D-E) by interacting with phosphorylated-Stat5 inside the nuclear (figure 1. F-I) and consequently activating Jak2/Stat 5 signaling pathway (figure 1. $\mathrm{J}-\mathrm{K})$. Rescue assays confirmed that Jak2/Stat 5 pathway is critical for the protumorigenic characterization of MGP to facilitate GC cell proliferation and inhibit apoptosis. 